\title{
Thermal Efficiency of a Solar Air-Heating Collector with a Metal Chip Absorber
}

\author{
Mirsoli O. Uzbekov and Azamat G. Tukhtasinov* \\ Ferghana Polytechnic Institute \\ Ferghan, Republic of Uzbekistan
}

Received 12.03.2020, received in revised form 21.04.2020, accepted 21.05.2020

\begin{abstract}
It is known that one of the main issues of heat transfer in complex designs of solar air-heating collectors is the problem of determining the coefficient of convective heat transfer in some parts of absorbers. The article studies the processes of heat transfer occurring in a solar air-heating collector with an absorber, which is a system of metal flow chips and a V-shaped surface. A description of the design of a solar air-heating collector with a metal chip absorber is given. The absorber allows us to increase the contact surface between the coolant and the absorber. The experimental procedure and the results are presented. The experiments have shown that within the range of $850-950 \mathrm{~W} / \mathrm{m}^{2}$ of direct incident solar radiation density, the average heating of coolant is $17.5^{\circ} \mathrm{C}$; within the range of $650-$ $750 \mathrm{~W} / \mathrm{m}^{2}$, it is $14.1^{\circ} \mathrm{C}$; within the range of $450-550 \mathrm{~W} / \mathrm{m}^{2}$, it measures $10.1{ }^{\circ} \mathrm{C}$. The maximum coolant heating is $27^{\circ} \mathrm{C}$ which is equal to the output collector temperature $60^{\circ} \mathrm{C}$. The average heating of the coolant is $14{ }^{\circ} \mathrm{C}$, which corresponds to the average output temperature of $45^{\circ} \mathrm{C}$. According to the data obtained in the experiment, empirical formulas are concluded. They are presented in the form of the dependence $\mathrm{Nu}=\mathrm{f}(\mathrm{Re})$, it is aimed at determining the heat transfer coefficient of flow metal chips and V-shaped absorbers of a solar air-heating collector. The dependence of the absorbers heat transfer from metal flow chips and the V-shaped surface of the solar air-heating collector on the Re numbers within the range of $103 \div 104$ is shown. A formula is derived that allows us to compare the effectiveness of absorbers of various types with a flat absorber of a solar air-heating collector.
\end{abstract}

Keywords: renewable energy sources, solar energy, solar air-heating collectors, absorber, efficiency, heat transfer, thermal efficiency.

Citation: Uzbekov M.O., Tukhtasinov A.G. Thermal efficiency of a solar air-heating collector with a metal chip absorber, J. Sib. Fed. Univ. Eng. \& Technol., 2020, 13(6), 712-720. DOI: 10.17516/1999-494X-0260

(C) Siberian Federal University. All rights reserved

This work is licensed under a Creative Commons Attribution-Non Commercial 4.0 International License (CC BY-NC 4.0).

* Corresponding author E-mail address: education.fer@mail.ru; a.tuxtasinov@ferpi.uz 


\title{
Тепловая эффективность солнечного
}

\author{
воздухонагревательного коллектора \\ с металлическим стружечным абсорбером
}

\author{
М.О. Узбеков, А.Г. Тухтасинов \\ Ферганский политехнический институт \\ Республика Узбекистан, Фергана
}

\begin{abstract}
Аннотация. Известно, что одним из основных вопросов теплообмена в сложных конструкциях солнечного воздухонагревательного коллектора считается проблема определения коэффициента конвективного теплообмена на отдельных участках абсорбера. В статье исследованы процессы теплообмена, протекающие в солнечном воздухонагревательном коллекторе с абсорбером, представляющем собой систему металлических сливных стружек и V-образную поверхность. Приводится описание конструкции солнечного воздухонагревательного коллектора с металлическим стружечным абсорбером, позволяющей увеличить контактную поверхность между теплоносителем и абсорбером, методика и результаты эксперимента. Эксперименты показали, что в диапазоне плотности прямой падающей радиации солнечного излучения $850-950 \mathrm{BT} / \mathrm{M}^{2}$ средний нагрев теплоносителя составляет $17,5^{\circ} \mathrm{C}$, в диапазоне $650-750 \mathrm{BT} / \mathrm{m}^{2}-$ $14,1{ }^{\circ} \mathrm{C}$, в диапазоне $450-550 \mathrm{BT} / \mathrm{M}^{2}-10,1{ }^{\circ} \mathrm{C}$. Максимальный нагрев теплоносителя $27{ }^{\circ} \mathrm{C}$, равный выходной температуре с коллектора $60{ }^{\circ} \mathrm{C}$, средний нагрев теплоносителя $14{ }^{\circ} \mathrm{C}$, что соответствует выходной средней температуре $45{ }^{\circ} \mathrm{C}$. На основании полученных данных в экспериментальных исследованиях получены эмпирические формулы в виде зависимости $\mathrm{Nu}=\mathrm{f}(\mathrm{Re})$ для определения величины коэффициента теплоотдачи сливных металлических стружек и V-образных абсорберов солнечного воздухонагревательного коллектора. Показаны зависимости теплоотдачи абсорберов из металлической сливной стружки и V-образной поверхности солнечного воздухонагревательного коллектора от чисел Re в диапазоне $10^{3} \div 10^{4}$. Выведена формула, позволяющая сравнить эффективность абсорберов различного типа с плоским абсорбером солнечного воздухонагревательного коллектора.
\end{abstract}

Ключевые слова: возобновляемые источники энергии, солнечная энергия, солнечные воздухонагревательные коллекторы, абсорбер, эффективность, теплоотдача, тепловая эффективность.

Цитирование: Узбеков, М.О. Тепловая эффективность солнечного воздухонагревательного коллектора с металлическим стружечным абсорбером / М.О. Узбеков, А.Г. Тухтасинов // Журн. Сиб. федер. ун-та. Техника и технологии, 2020. 13(6). С. 712-720. DOI: 10.17516/1999-494X-0260

Введение. Солнечная энергия является свободно доступным, бесконечным, экологически чистым энергоресурсом. [1]. Одно из перспективных направлений возобновляемой энергетики - прямое получение экологически чистого тепла для системы кондиционирования, сушки сельскохозяйственной продукции с преобразованием солнечной радиации $[2,3]$. Среди различных типов солнечных тепловых установок солнечные воздухонагревательные коллекторы (СВК) широко используют из-за более низкой стоимости и простоты конструкции $[4,5]$. Существует много вариантов СВК для нагрева воздуха, преимущество имеют СКВ с плоским гладким абсорбером благодаря простоте конструкции, простоте в эксплуатации, низкому техническому обслуживанию. Основные типы СВК с плоским абсорбером $[6,7]$ обладают низкими тепловыми характеристиками из-за меньшего теплоусвоения путем прохождения воздуха через канал в виде конвективного теплообмена. Полезное тепло из СВК может быть извлечено 
естественным или принудительным способом. Обычный СВК состоит из остекления, поглотителя, нижней плиты и изоляционных материалов $[8,9]$. Поток воздуха проходит над или под пластиной поглотителя для сбора тепла, иногда с обеих сторон. Основная задача при использовании воздуха в качестве теплоносителя - повышение коэффициента теплоотдачи. Поэтому необходимо улучшить тепловой КПД солнечного воздухонагревателя, применяя подходящий способ повышения теплопередачи. Пути повышения теплопередачи солнечных воздухонагревателей, рассмотренных авторами в [10], требуют значительного капиталовложения для создания эффективных абсорберов [11]. Из-за этого также остается актуальным вопрос снижения экономических затрат на изготовление эффективных абсорберов и солнечных воздухонагревателей.

Методы и материалы. На рис. 1 представлена конструкция солнечного воздухонагревательного коллектора (СВК) с металлическим стружечным абсорбером. СВК содержит легкий теплоизолированный корпус из трехстенной двухкамерной пластмассы 1 , на которой установлен каркас 2 для уплотнения крышки 8 и корпуса 1, три перегородки установлены 3 поперек относительно потока воздуха на дне корпуса, абсорбер из V-образного волнистого металлического листа, состоящий из ряда V-образных форм 4, установлен поперек каналов относительно движения воздуха, укрепленный на перегородке каркас 5 из алюминиевого профиля, каркас металлической сетки установлен на металлическом листе V-образной формы, металлическая сетка 6, натянутая в каркасе, абсорбер из нержавеющих стальных сливных стружек 7, сливные стружки установлены поперек относительного движения воздуха, они равномерно распределены на металлической сетке, крышка 8 солнечного воздухонагревателя оснащена прозрачным покрытием 9, выполнена из стекла, подводящий 10 и отводящий 11 воздух патрубки, вентилятор 12 для нагнетания воздуха через подводящий патрубок.

Геометрические размеры разработанной конструкции: длина коллектора 1,5 м, высота коллектора 0,072 м, ширина коллектора 0,5 м. Площадь абсорбера V-образной формы 0,7 м², площадь абсорбера из металлической стружки $0,725 \mathrm{~m}^{2}$.

СВК работает следующим образом: воздух, нагнетаемый вентилятором 12 , поступает в пространство через подводящий патрубок 10 в нижнюю часть абсорбера в канал, состоящий из V-образного волнистого металлического листа 4 и дна коллектора 1. Воздушный поток, омывая тыльную сторону абсорбера из V-образного волнистого металлического листа 4, обтекает перегородки 3. Далее воздух поступает через воздушный канал в пространство между верхней частью абсорбера из V-образного волнистого металлического листа и прозрачным покрытием 9, воздух омывает абсорбер из нержавеющих стальных сливных стружек 7 и верхнюю часть абсорбера из V-образного волнистого металлического листа. Далее воздух через отводящие патрубки 11 выходит из коллектора.

Воздух, обтекая перегородки 3, установленные в дне корпуса 1, многократно меняет свое направление, искусственно увеличивая свой путь прохождения через СВК.

Экспериментальные исследования СВК проводили в следующем порядке: при фиксированном значении угла наклона СВК $45^{\circ}$, при фиксированном расходе воздуха, расход воздуха варьировался за счет изменения напряжения вентилятора, при различных режимах массового расхода воздуха и солнечной радиации, с предварительным включением регистрирующих приборов. 

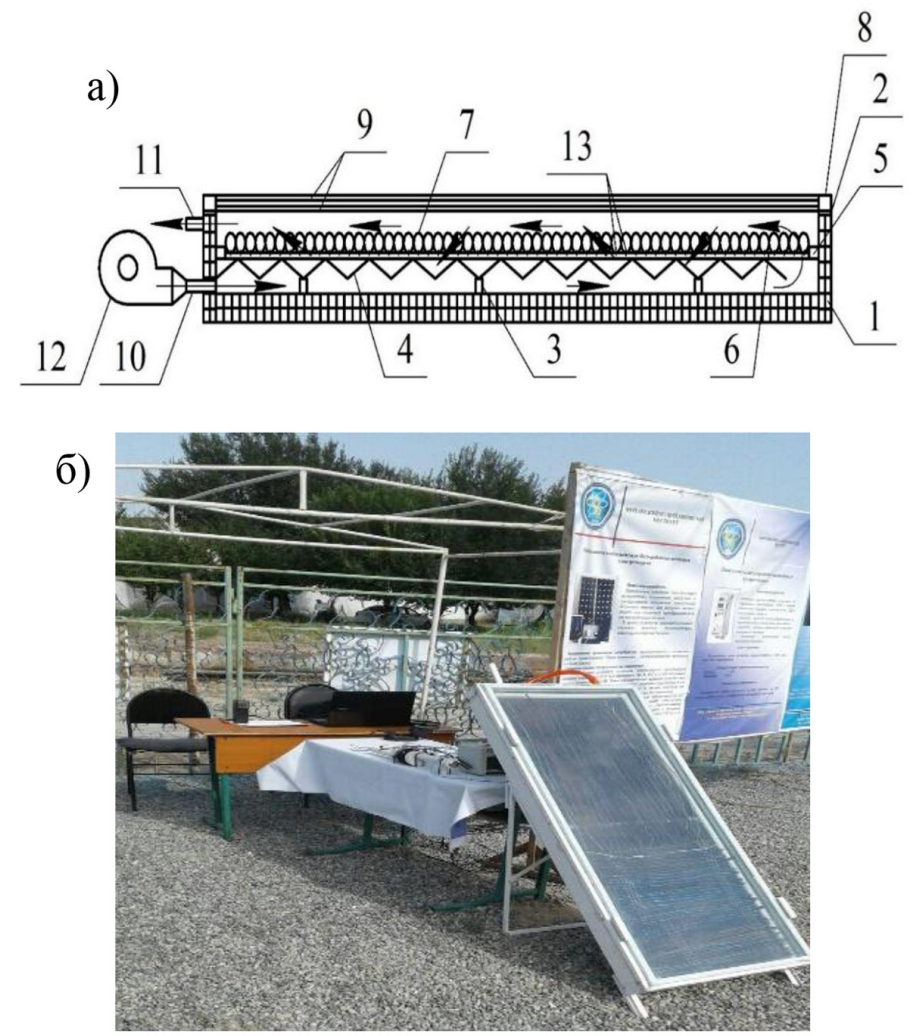

Рис. 1. Принципиальная схема (а) и обший вид (б) экспериментального СВК

Fig. 1. Schematic diagram (a) and general view (б) of the experimental ICS

В табл. 1 приводится пример протокола испытания, разработанного СВК.

На основании полученных в опытах результатов рассчитана теплообменная эффективность теплопередачи СВК. Формулы теплообмена на отдельных абсорберах представляются в виде

$$
N u=A(\mathrm{Re})^{n} .
$$

Представление формул теплообмена в таком виде соответствует положениям теории подобия, которая для жидкостей и газов записывается в общем виде:

$$
N u=A(\operatorname{Re} \operatorname{Pr})^{n} .
$$

Если считать, что для газов (воздуха) $P_{r} \approx 1$, формула (2) преобразуется в формулу (1). Обработка полученных экспериментальных данных позволила получить для V-образного абсорбера формулу теплообмена:

$$
N u_{V}=0,014 \mathrm{Re}^{0,83},
$$

для стружечного абсорбера

$$
N u_{c m}=0,02 \operatorname{Re}^{0,82} .
$$


Таблица 1. Протокол испытания солнечного воздухонагревательного коллектора

Table 1. Solar Hot Air Collector Test Report

\begin{tabular}{|c|c|c|c|c|c|c|c|c|}
\hline \multicolumn{9}{|c|}{ Протокол испытания от 15.09.2019 года } \\
\hline \multirow[t]{2}{*}{ № } & \multirow[t]{2}{*}{$\mathrm{G}$, кг/с } & \multirow[t]{2}{*}{$\mathrm{t}_{\mathrm{BX}},{ }^{\circ} \mathrm{C}$} & \multirow[t]{2}{*}{$\mathrm{t}_{\text {вых }},{ }^{\circ} \mathrm{C}$} & \multicolumn{2}{|c|}{$\begin{array}{c}\text { Солнечная радиация } \\
\text { Вт/м }{ }^{2}\end{array}$} & \multirow[t]{2}{*}{$\mathrm{t}_{2},{ }^{\circ} \mathrm{C}$} & \multirow[t]{2}{*}{$\mathrm{t}_{3},{ }^{\circ} \mathrm{C}$} & \multirow[t]{2}{*}{$\mathrm{t}_{4},{ }^{\circ} \mathrm{C}$} \\
\hline & & & & Прямая & Диффузная & & & \\
\hline 1 & 0,0025 & 33,8 & 60,8 & 803 & 100 & 41,9 & 52,5 & 53,2 \\
\hline 2 & 0,0033 & 33,8 & 60,3 & 805 & 100 & 41,75 & 51,5 & 52,8 \\
\hline 3 & 0,0061 & 32,8 & 57,8 & 804 & 95 & 40,3 & 49,6 & 50,8 \\
\hline 4 & 0,009 & 33,1 & 57,1 & 806 & 95 & 40,3 & 48,4 & 50,4 \\
\hline 5 & 0,015 & 32,8 & 54,3 & 796 & 100 & 39,3 & 46,1 & 48,3 \\
\hline 6 & 0,0233 & 33,1 & 53,1 & 796 & 103 & 39,1 & 44,9 & 47,5 \\
\hline 7 & 0,0284 & 33,1 & 50,1 & 796 & 103 & 38,2 & 43,5 & 45,3 \\
\hline 8 & 0,0323 & 33,2 & 48,2 & 800 & 100 & 37,7 & 42,1 & 44 \\
\hline 9 & 0,0387 & 33,1 & 45,7 & 798 & 102 & 36,9 & 40,8 & 42,2 \\
\hline 10 & 0,0438 & 33,4 & 44,6 & 798 & 102 & 36,8 & 39,9 & 41,5 \\
\hline 11 & 0,0497 & 33,3 & 43,4 & 800 & 102 & 36,3 & 39,2 & 40,6 \\
\hline
\end{tabular}

При расчетах теплообмена за определяющий размер для V-образного абсорбера эквивалентный диаметр канала воздухонагревателя принят равным 0,026 м.

$$
\begin{aligned}
& \alpha=\frac{G c_{p}\left(t^{\prime \prime}-t^{\prime}\right)}{F \Delta t}=\frac{0,0493 \cdot 2,4 \cdot 10^{3}}{0,9 \cdot 5}=26,3 \\
& N u=\frac{\alpha d}{\lambda}=\frac{26,3 \cdot 0,026}{0,027}=25,3
\end{aligned}
$$

В табл. 2 приведены результаты расчета теплообмена V-образного абсорбера.

$$
\begin{aligned}
& \alpha=\frac{G c_{p}\left(t^{\prime \prime}-t^{\prime}\right)}{F \Delta t}=\frac{0,0493 \cdot 12,4 \cdot 10^{3}}{0,9 \cdot 9,9}=68,5, \\
& N u=\frac{\alpha d}{\lambda}=\frac{68,5 \cdot 0,015}{0,027}=38 .
\end{aligned}
$$

В табл. 3 приведены результаты расчета теплообмена стружечного абсорбера.

Результаты расчета теплоотдачи по (3) и (4) V-образного и металлического стружечного абсорбера солнечного воздухонагревательного коллектора представлены на рис. 2.

Обобщенная формула эффективности теплоотдачи абсорбера солнечного воздухонагревательного коллектора получена путем применения метода суперпозиции. Смысл метода суперпозиции заключается в том, что каждая из поверхностей абсорбера вносит свой определенный вклад в общий процесс переноса теплоты в солнечном воздухонагревательном коллекторе. Так как поток воздуха последовательно проходит отдельные абсорберы, то принимаем, что скорость в участках постоянна, $w_{1}=w_{2}=w_{3}=w_{\text {гл}}$, диаметры каналов

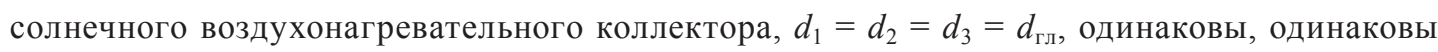
и значения чисел $\operatorname{Re}_{1}=\operatorname{Re}_{2}=\operatorname{Re}_{3}=\mathrm{Re}_{\text {гл }}$ в каналах солнечного воздухонагревательного коллектора. 
Таблица 2. Результаты расчетов теплообмена V-образного абсорбера

Table 2. Results of calculations of heat transfer of a V-shaped absorber

\begin{tabular}{|c|c|c|c|c|}
\hline \multirow{2}{*}{$\mathrm{N}$} & \multirow{2}{*}{$\operatorname{Re}_{2 л}$} & \multicolumn{3}{|c|}{ Теплообмен V-образного абсорбера } \\
\cline { 3 - 5 } & & $\begin{array}{c}\text { Экспериментальные } \\
\text { данные }\end{array}$ & $N u_{V}=0,014 R e^{0,83}$ & Погрешность, \% \\
\hline 1 & 9269 & 25,3 & 27,46 & 7,8 \\
\hline 2 & 8222 & 23,2 & 24,86 & 6,7 \\
\hline 3 & 7264 & 20,9 & 22,43 & 6,8 \\
\hline 4 & 6066 & 18,4 & 20 & 8 \\
\hline 5 & 5373 & 16,5 & 17,46 & 5,5 \\
\hline 6 & 4333 & 14,1 & 14,61 & 3,5 \\
\hline
\end{tabular}

При расчетах теплообмена для стружечного абсорбера за определяющий размер принят диаметр стружки 0,015 м.

Таблица 3. Результаты расчета теплообмена стружечного абсорбера

Table 3. Results of calculating the heat transfer of the metalline fillings absorber

\begin{tabular}{|c|c|c|c|c|}
\hline \multirow{2}{*}{$\mathrm{N}$} & \multirow{2}{*}{$e_{2 л}$} & \multicolumn{3}{|c|}{ Теплообмен стружечного абсорбера } \\
\cline { 3 - 5 } & 9269 & $\begin{array}{c}\text { Экспериментальные } \\
\text { данные }\end{array}$ & $N u_{\mathrm{cт}}=0,02 \operatorname{Re}^{0,82}$ & Погрешность, \% \\
\hline 1 & 8222 & 38 & 35,8 & 6,14 \\
\hline 2 & 7264 & 33,7 & 32,46 & 3,8 \\
\hline 3 & 6066 & 27,9 & 29,32 & 4,8 \\
\hline 4 & 5373 & 23,7 & 25,9 & 8,5 \\
\hline 5 & 4333 & 21,5 & 22,89 & 6,1 \\
\hline 6 & & 18,1 & 19,19 & 5,7 \\
\hline
\end{tabular}

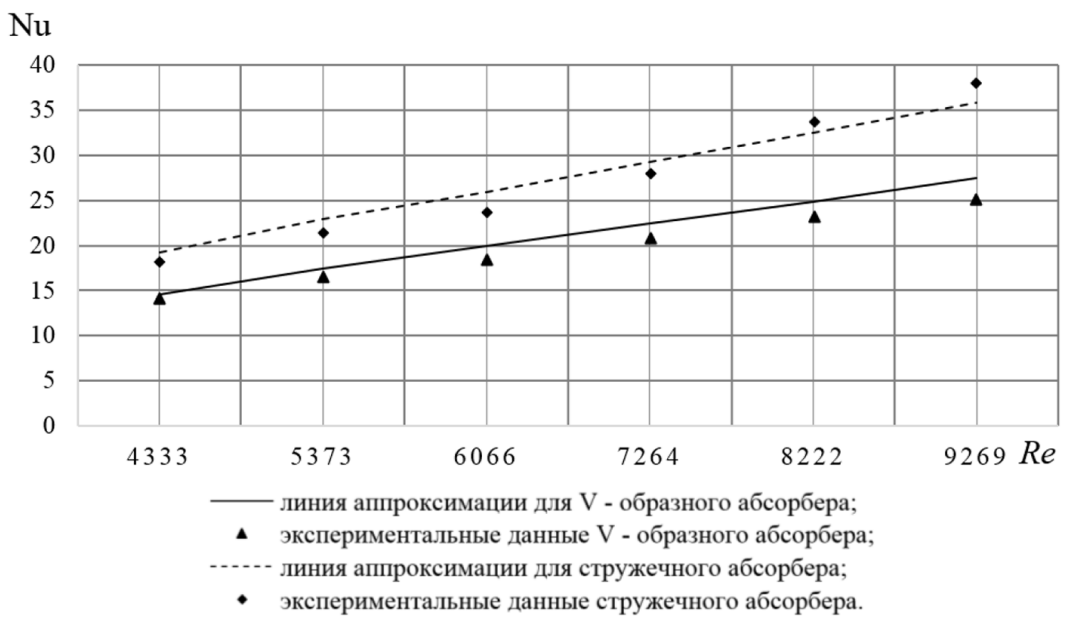

Рис. 2. Зависимость теплоотдачи абсорберов из металлической сливной стружки и V-образной поверхности солнечного воздухонагревательного коллектора от чисел $R e$

Fig. 2. The dependence of the heat transfer of absorbers from metal drainage chips and the V-shaped surface of the solar air heater collector on the numbers $R e$ 
В общем случае формула теплообмена имеет вид (1), а для V-образного абсорбера солнечного воздухонагревательного коллектора формула эффективности теплоотдачи имеет вид

$$
\frac{N u_{V}}{N u_{2 n}}=\frac{A_{V} \operatorname{Re}^{n_{V}}}{C \operatorname{Re}_{2 n}^{m}}=\frac{A_{V}}{C} \operatorname{Re}^{n_{V}-m} .
$$

Для стружечного абсорбера солнечного воздухонагревательного коллектора

$$
\frac{N u_{c m}}{N u_{2 n}}=\frac{A_{c m} \operatorname{Re}^{n_{c m}}}{C \operatorname{Re}_{2 n}^{m}}=\frac{A_{c m}}{C} \operatorname{Re}^{n_{c m}-m} .
$$

Общая формула эффективности теплообмена солнечного воздухонагревательного коллектора на основании вышеуказанного имеет вид

$$
\frac{N u}{N u_{2 n}}=\frac{F_{V} N u_{2 n} \frac{A_{V}}{C} \operatorname{Re}_{2 n}^{n_{V}-m}+F_{c m} N u_{2 n} \frac{A_{c m}}{C} \operatorname{Re}_{2 n}^{n_{c m}-m}}{F N u_{2 n}}=\frac{1}{2} \cdot\left[\frac{A_{V}}{C} \operatorname{Re}^{n_{V}-m}+\frac{A_{c m}}{C} \operatorname{Re}^{n_{c m}-m}\right],
$$

где $n_{V}, n_{\mathrm{c \tau}}, m-\operatorname{tg}$ угла наклона прямой линии, находятся из экспериментальных данных по оси абсцисс для каждого абсорбера $\lg \operatorname{Re}$ (для гладкого абсорбера $m=0,80$, для V-образного абсорбера $n_{V}=0,83$, для металлического стружечного абсорбера $\left.n_{\text {ст }}=0,82\right) ; A_{V}, A_{\mathrm{cr}}, C$ - постоянные числовые коэффициенты, находятся из экспериментальных данных (для гладкого абсорбера $C=0,018$, для $\mathrm{V}$-образного абсорбера $A_{V}=0,014$, для металлического стружечного абсорбера $\left.A_{\text {ст }}=0,02\right) ; F_{V}, F_{\text {ст }}$, $F$ - площади абсорберов, $\mathrm{m}^{2} ; N u_{v}, N u_{\text {ст }}, N u_{\text {гл }}$ числа Нуссельта для каждого абсорбера.

В табл. 4 приведена эффективность абсорберов относительно гладкой поверхности.

Анализ результатов. Из данных табл. 4 следует что теплопередающая эффективность 1-го абсорбера практически не меняется, однако за счет повышения теплопередающей эффективности 2-го абсорбера при числах $R e$ от 9269 до 4333 увеличивается эффективность СВК с металлической стружкой. Данная закономерность, полученная в результате экспериментов, объясняет тот факт, что при снижении чисел и, как следствие, скорости воздушного потока происходит повышение колебаний температуры.

Эффективность достигается за счет увеличения времени прохождения потока через 2 абсорбер, а также вследствие высокой эффективности теплообмена на поверхности металлической стружки.

Таблица 4. Эффективность абсорберов относительно плоской поверхности

Table 4. Efficiency of absorbers relatively flat surface

\begin{tabular}{|c|c|c|c|c|c|c|}
\hline$R e$ & 9269 & 8222 & 7264 & 6066 & 5373 & 4333 \\
\hline$\frac{N u}{N u_{\text {гл }}}$ & 1,17 & 1,17 & 1,17 & 1,16 & 1,16 & 1,16 \\
\hline$\frac{N u_{1}}{N u_{\text {гл }}}$ & 1,02 & 1,02 & 1,01 & 1,0 & 1,0 & 1,0 \\
\hline$\frac{N u_{2}}{N u_{\text {гл }}}$ & 1,33 & 1,33 & 1,33 & 1,32 & 1,32 & 1,32 \\
\hline
\end{tabular}


Невысокая тепловая эффективность теплопередачи первого абсорбера относительно второго объясняется тем, что первый абсорбер играет роль подогрева наружного воздуха в первой ступени. Кроме того, низкая тепловая эффективность первого абсорбера связана с недостаточным поглощением солнечной энергии профилированным листом по сравнению с высокопоглощающей эту энергию поверхностью металлической стружки.

\section{Вывод}

Использование стружечного теплоприемника СВК повышает тепловую эффективность на 15 \% относительно V-образного теплоприемника.

\section{Список литературы / References}

[1] Renewables 2007 Global Status Report. www.ren21.net.

[2] Попель О.С. Возобновляемые источники энергии: роль и место в современной и перспективной энергетике. Рос. хим. журнал, 2008, LII(6). [Popel O.S. Renewable energy sources: role and place in modern and promising energy. Grew up. chem. g. (Zh. Ros. Chem. Society named after D.I. Mendeleev), 2008, LII(6) (in Russian)]

[3] Kuchkarov, A.A., Khaitmukhamedov, A.E., Shukurov, A.O. et al. Calculation of Thermal and Exergy Efficiency of Solar Power Units with Linear Radiation Concentrators. Appl. Sol. Energy 2020, 56, 42-46. https://doi.org/10.3103/S0003701Х20010089.

[4] Виссарионов В.И., Золотов Л.А. Экологические аспекты возобновляемых источников энергии. М.: Изд-во МЭИ, 2008. 156 с. [Vissarionov V.I., Zolotov L.A. Ecological aspects of renewable energy sources. Moscow: MPEI Publishing House, 2008. 156 p. (in Russian)]

[5] Avezov R.R., Avezova N.R., Matchanov N.A., Suleimanov Sh.I., Abdukadirova R.D. History and State of Solar Engineering in Uzbekistan. Applied Solar Energy, 2012, 48(1), 14-19.

[6] Uzbekov M.O. Thermal balance of the solar air heater with a heat sink of metal shavings. International Journal of Advanced Research in Science, Engineering and Technology, 2019, 6(5), 9246-9254

[7] Oussama Touaba, Mohamed Salah Ait Cheikh, Mohamed El-Amine Slimani et al. Experimental investigation of solar water heater equipped with a solar collector using waste oil as absorber and working fluid. Solar Energy, 2020, 199(15), 630-644.

[8] Узбеков М.О. и др. Исследование термического сопротивления солнечного воздухонагревателя с металлической стружкой. Энергосбережение и водоподготовка, 2019, 4, 29-33. [Uzbekov M.O. et al. Investigation of the thermal resistance of a solar air heater with metal shavings. Energy saving and water treatment, 2019, 4, 29-33 (in Russian)]

[9] Avezov R.R. and Lutpullaev S.L., The State of Art, Trends and Problems for Applying the Renewable Energy Sources in Uzbekistan, "Fizika v Uzbekistane". Materialy konf., posvyashchennoi "Godu fiziki-2005" (Proc. Conf. "Physics in Uzbekistan" Dedicated to "Solar Year-2005"), Tashkent: AN RUz, Sept. 27-28, 2005, pp. 119-123.

[10] Uzbekov M.O. Possibilities of increasing the efficiency of the heat receiver of ics from metal shavings. Computational nanotechnology, 2019, 2, 138-140.

[11] Узбеков М.О., Тухтасинов А.Г. Измерения температуры нагрева абсорбера солнечного воздухонагревательного коллектора. Universum: Технические науки: электрон. на- 
учн. журн. 2020, 6(75). URL: http://7universum .com/ru/tech/archive/item/9604 [Uzbekov M.O., Tukhtasinov A.G. Measurements of the heating temperature of the absorber of the solar air heating collector. Universum: Technical sciences: electron. scientific. zhurn. 2020, 6 (75) (in Russian)] 